\title{
Mesenteric venous thrombosis and antithrombin III deficiency
}

\author{
C WILSON, $\dagger$ I D WALKER,* J F DAVIDSON,* C W IMRIE $\dagger$ \\ From the $\dagger$ Division of Surgery and ${ }^{*}$ Department of Haematology, Royal Infirmary, Glasgow
}

SUMMARY Of the 123 patients with acute mesenteric infarction treated over the past 12 years, 16 $(13 \%)$ had mesenteric venous thrombosis. Eight of the patients with mesenteric venous thrombosis survived the initial episode; two have since died. The remaining six patients were studied for evidence of haemostatic deficiencies or abnormalities. Antithrombin III deficiency, which is known to be associated with recurrent venous thrombosis, was found in three patients. It is recommended that all patients with mesenteric venous thrombosis should be screened for antithrombin III deficiency as treatment with courmarin anticoagulants may be indicated, providing effective prophylaxis against further thrombotic episodes.

Mesenteric venous thrombosis accounts for over 7\% of patients with mesenteric infarction. ${ }^{1}$ Conditions predisposing to mesenteric venous thrombosis include portal hypertension, intra-abdominal sepsis, haematological disorders (such as polycythaemia rubra vera), neoplasms, surgery (particularly splenectomy) and use of oral contraceptives. Most patients have primary mesenteric venous thrombosis, where no underlying cause is found.

Abnormalities of the haemostatic mechanism are increasingly recognised: antithrombin III (ATIII), protein $\mathrm{C}$, and protein $\mathrm{S}$ deficiencies and plasminogen abnormalities have been described in recurrent venous thrombosis. ${ }^{2-5}$ Mesenteric venous thrombosis was first reported in association with familial ATIII deficiency in $1975 .^{6}$

\section{Patients and methods}

We reviewed the records of all 123 patients with acute mesenteric infarction presenting between January 1973 and August 1984, of whom $16(13 \%)$ had mesenteric venous thrombosis. Thirteen of these patients were diagnosed at laparotomy and three at necropsy. Mean age was 55 years (range 32-74); eight patients $(50 \%)$ were male. Mesentric venous thrombosis was considered to be secondary to other underlying disease in five patients $(31 \%)$, four of whom died. Three of these patients had underlying gastrointestinal carcinoma, one patient was receiving stilboestrol for prostatic carcinoma, and the fifth patient developed superior and inferior mesenteric vein and splenic vein

Accepted for publication 26 March 1987 thrombosis secondary to acute necrotising pancreatitis. In the remaining patients no recognised underlying cause of thrombosis could be implicated although one developed mesenteric venous thrombosis three weeks after giving birth and another had evidence of secondary polycythaemia on admission; both factors were likely to have been of importance. Thrombosis was not associated with use of oral contraceptives. Three patients $(19 \%)$ had a history of thrombotic episodes, one had had mesenteric venous thrombosis nine years before and the other two each had had an episode of deep vein thrombosis.

Eight patients $(50 \%)$ survived the initial episode, and two of them have since died. The remaining six patients were studied for evidence of haemostatic deficiencies or abnormalities.

ATIII and plasminogen antigen were measured by radial immunodiffusion (NOR and $M$ Partigen plates, Behring Diagnostics). ATIII (functional) was measured by amidolytic assay ${ }^{7}$ and protein $\mathrm{C}$ by a modified enzyme linked immunosorbent assay (ELISA) method (Boehringer Mannheim). The func- N tional assay of plasminogen was done according to the amidolytic method of Friberger. ${ }^{8}$ The normal ranges for all tests were established in over $\mathbf{4 0}$ healthy volunteers.

\section{Case reports}

CASE 1

A 32 year old man who presented with abdominal pain in 1984 was found at laparotomy to have venous congestion of the entire small bowel with multiple $\mathbb{D}$ infarcted areas. The spleen appeared enlarged but the 
Table Results of haemostatic assessment

\begin{tabular}{|c|c|c|c|c|c|}
\hline \multirow[b]{2}{*}{ Case No } & \multicolumn{2}{|l|}{ Antithrombin III } & \multirow{2}{*}{$\frac{\text { Protein C }}{\substack{\text { Immunological } \\
(60-133 \%)}}$} & \multicolumn{2}{|l|}{ Plasminogen } \\
\hline & $\begin{array}{l}\text { Immunological } \\
(29-42 \mathrm{mg} / \mathrm{dl})\end{array}$ & $\begin{array}{l}\text { Amidolytic } \\
(85-129 \%)\end{array}$ & & $\begin{array}{l}\text { Immunological } \\
(0.09-0.15 \mathrm{~g} / \mathrm{l})\end{array}$ & $\begin{array}{l}\text { Amidolytic } \\
(73-104 \%)\end{array}$ \\
\hline $\begin{array}{l}1 \\
2 \\
3 \\
4 \\
5 \\
6\end{array}$ & $\begin{array}{l}23 \\
20 \\
25 \\
36 \\
29 \\
34\end{array}$ & $\begin{array}{r}69 \\
61 \\
72 \\
107 \\
89 \\
116\end{array}$ & $\begin{array}{r}50^{*} \\
59 \\
113 \\
100 \\
87\end{array}$ & $\begin{array}{l}0.08 \\
0.10 \\
0.14 \\
0.13 \\
0.10\end{array}$ & $\begin{array}{r}87 \\
127 \\
113 \\
102\end{array}$ \\
\hline
\end{tabular}

* Patient receiving warfarin.

liver was normal with no stigma of portal hypertension. The infarcted bowel was resected and the liver biopsied. Histological examination of the resected bowel confirmed mesenteric venous thrombosis. The liver biopsy specimen showed a chronic inflammatory cell infiltrate in the portal tracts and moderate fatty change.

Seven days postoperatively the ATIII activity was assayed and found to be $56 \%$ of normal. ATIII concentrate (2000 units/day) was given to maintain plasma ATIII activity above $80 \%$. Liver function tests, one stage prothrombin time, kaolin cephalin clotting time, and thrombin time were all normal. Warfarin was subsequently given and continues to be given at the time of writing, as ATIII activity remained persistently low on follow up (table). Screening his mother and two brothers showed normal ATIII activity $(100 \%, 100 \%$, and $103 \%$ of normal, respectively).

\section{CASE 2}

A 66 year old man underwent resection of a short segment of terminal ileum for venous infarction in 1979. Routine haematology at that time was normal but haemostatic assessment was not carried out. He developed an ischaemic finger three years later. Arch aortography showed a likely brachial artery occlusion with normal lower arm and digital vessels. Haematological investigation showed ATIII deficiency (ATIII $43 \%$ of normal), essential thrombocythaemia (platelets $711 \times 10^{9} / 1$ ), and a coagulopathy with reduced factors II $(43 \%)$, VII $(46 \%)$, IX $(64 \%)$, and $X(41 \%)$. Liver function tests yielded normal results except for gamma-glutamyl transpeptidase and lactate dehydrogenase activities which showed a persistent, mild increase. Subsequent liver biopsy yielded normal results. Treatment with radiophosphorus (40 MBQ) resulted in satisfactory control of the thrombocythaemia but ATIII activity remained persistently low on follow up (table). Screening his two siblings showed that they had normal ATIII activity ( $94 \%$ and $97 \%$ of normal).
CASE 3

A 76 year old man admitted in 1974 was found at laparotomy to have venous congestion of the entire small bowel, which was maximal in the mid jejunum. The bowel was viable and therefore not resected. He was not investigated postoperatively but treated empirically with heparin, making an uneventful recovery. He has since had one episode of deep vein thrombosis. As part of this survey he was studied for haemostatic abnormality, which showed mild ATIII deficiency (ATIII 72\% of normal). Estimation of protein $\mathrm{C}$, plasminogen, and coagulation screen and liver function tests all yielded normal results.

Three other survivors were reviewed and screened for ATIII, protein C, and plasminogen abnormalities two, three, and 11 years, respectively, following their mesenteric venous thrombosis. None has had further thrombotic episodes and all had normal haemostatic values at the time of writing.

\section{Discussion}

Antrithrombin III is the most important naturally occurring antithrombin. It inhibits thrombin and also factor $\mathrm{Xa}$, and thus regulates thrombin formation. ATIII is an $\alpha-2$-globulin with a molecular weight of around 65000 . It is synthesised in the liver and has a narrow physiological range in plasma, values below this range being associated with an increased tendency to venous thrombosis. Deficiency of ATIII may result from impaired or faulty synthesis in congenital or acquired disorders, increased consumption or catabolism, loss from the intravascular compartment, or from unknown causes. ${ }^{9}$

Hereditary ATIII deficiency is usually transmitted as an autosomal dominant and such families have a high incidence of venous thrombosis. Over $85 \%$ of those aged 50 or older have had at least one thrombotic episode, often precipitated by pregnancy, parturition, surgery, trauma, or use of oral contraceptives. ${ }^{9}$ The mesenteric veins are a rare but characteristic site of thrombosis in familial ATIII 
deficiency, affecting $8 \%$ of reported cases. ${ }^{10}$

Biochemical and haematological evidence suggested liver dysfunction as a possible cause for ATIII deficiency in case 2. ATIII concentrations may be reduced in chronic liver disease, but because of a simultaneous decrease in the procoagulants, there may be no increased risk of thrombosis. ${ }^{9}$ In case 2 , despite the reduced procoagulants, the ATIII deficiency and thrombocythaemia presumably favoured thrombosis.

The aetiology of ATIII deficiency in the two other patients remains unclear, although in case 1 there may have been a liver abnormality. We were unable to show the deficiency in the other family members who were available for testing. Patients did not have abnormal plasminogen values and none had protein $\mathrm{C}$ deficiency, although this is thought to be commoner than ATIII deficiency. ${ }^{11}$

Moderate ATIII deficiency seems to have been a factor in three of the six survivors from mesenteric venous thrombosis tested, and it may explain the mechanism of thrombosis in some patients who would previously have been classified as having primary mesenteric venous thrombosis. Familial ATIII deficiency is a recognised but rare cause of mesenteric venous thrombosis. Our study suggests that even acquired ATIII deficiency may be associated with this condition, and we would therefore recommend investigating all patients with mesenteric venous thrombosis for ATIII deficiency as soon as the condition is suspected. In the presence of clinically important thrombosis ATIII activity may be decreased due to ATIII consumption and therefore results in the acute phase of the illness should be interpreted with caution. ATIII activity may fall further in the postoperative period if the patient remains unwell due to infection, septicaemia, or disseminated intravascular coagulation. ${ }^{9}$ Careful follow up with repeated ATIII estimation is therefore essential to avoid mislabelling patients whose ATIII activity subsequently returns to normal. Families of those discovered to have ATIII deficiency should also be studied to exclude familial deficiency because of their very high risk of thrombotic disease.

Treatment with coumarin anticoagulants provides effective prophylaxis further thrombosis in ATIII deficiency ${ }^{12}$ and is indicated in familial cases, in those with a low ATIII activity associated with previous thrombotic episodes, and particularly in situations likely to predispose to thrombosis such as surgery. In pregnancy, due to the teratogenic risks associatede. with coumarin anticoagulants, prophylactic anti coagulation with heparin is preferable at least during? the first trimester. ${ }^{10}$ Immediately after surgery anti은 coagulation with heparin alone may be ineffective in preventing further thrombosis in patients with ATIIf deficiency. The concurrent administration of ATIIB concentrate and heparin permits immediate, full anti coagulation, although the doses of both require care $\vec{P}$ ful laboratory monitoring, ${ }^{12}$ which for ATIII, should be by a functional amidolytic assay. ${ }^{13}$

We thank Dr J Conkie and the staff of the Throm웡 bosis Research Laboratory for performing the assays ${ }_{0}$

\section{References}

1 Ottinger LW, Austen WG A study of 136 patients with meser teric infarction. Surg Gynecol Obstet 1967;124:251-61.

2 Egeberg $O$. Inherited antithrombin deficiency causing thrombophilia. Thrombosis Diath Haemorrh 1965;13:516-30.

3 Griffin JH, Evatt B, Zimmerman TS, Kleiss AJ, Wideman Deficiency of protein $\mathrm{C}$ in congenital thrombotic disease. J Cling Invest 1981;68:1370-3.

4 Aoki N, Moroi M, Sakata Y, Yoshida N, Matsuda M. Abnormalo plasminogen. A hereditary molecular abnormality found in a patient with recurrent thrombosis. J Clin Invest 1978;61: 1186-95.

5 Comp PC, Nixon RR, Cooper R, Esmon CT. Familial protein S deficiency is associated with recurrent thrombosis. J Clin Inves? 1984;74:2082-8.

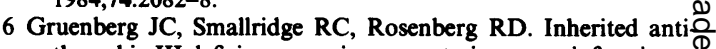
thrombin III deficiency causing mesenteric venous infarction: new clinical entity. Ann Surg 1975;181:791-4.

7 Abildgaard U, Lie M, Odegard OR. Antithrombin (heparin cof actor) assay with "new" chromogenic substrates (S-2238 and chromozym TH). Thromb Res 1977;11:549-53.

8 Friberger P, Knos M, Gustavsson S, Aurell L, Claeson G. Meth? ods for determination of plasmin, antiplasmin and plas = minogen by means of substrate S-2251. Haemostasis 1978;7:138-45.

9 Thaler E, Lechner K. Antithrombin III deficiency and throm boembolism. Clin Haematol 1981;10:369-90.

10 Anonymous. Familial antithrombin III deficiency [Editorial] Lancet 1983;i:1021-2.

11 Horellou MH, Conard J, Bertina RM, Samama M. Congenitab protein $\mathrm{C}$ deficiency and thrombotic disease in nine French? families. Br Med J 1984;289:1285-7.

12 Winter JH, Fenech A, Ridley W, et al. Familial antithrombin IIE. deficiency. $Q J$ Med 1982;51:373-95.

13 Mannucci PM, Boyer C, Wolf M, Tripodi A, Larrieu MJ. Treat ment of congenital antithrombin III deficiency with concen N trates. Br J Haematol 1982;50:531-5.

Requests for reprints to: Mr C Wilson, Department oP Surgery, Royal Infirmary, Glasgow G4 0SF, Scotland. 